\title{
Comparison of pain severity, satisfaction, and complications of proximal and distal forearm anesthesia in patients undergoing trans-palmar coronary angiography
}

\begin{abstract}
Objective: Trans-palmar access is a novel, safe, and feasible technique for coronary artery angiography wherein its appropriate anesthetic methods is still concerned. In this study, we aimed to evaluate the pain severity, satisfaction, and possible complications with local ulnar nerve anesthesia through both distal and proximal forearm in patients undergoing trans-palmar coronary angiography.
\end{abstract}

Methods: This was a randomized clinical trial performed on 60 patients who were candidates for trans-palmar coronary angiography. The patients were randomized into 2 equal groups as proximal and distal approaches (those who received the same dose of subcutaneous lidocaine $(2 \%)$ in the proximal and distal of forearm, respectively). Pain intensity at different times, duration of anesthesia, patient satisfaction, and occurrence of complications were evaluated.

Results: The mean age of the patients was $59.45 \pm 7.09$ years, and, of them, $34(61.8 \%)$ were men. Pain severity with the proximal anesthesia approach was significantly higher than that in the distal group at the time of puncture $(5.39 \pm 0.73$ vs. $2.30 \pm 0.60, p=0.001)$. Over time and immediately after the procedure and at discharge, the mean pain severity in the proximal group was significantly less than in the distal group $(p<0.050)$. The proximal group also had a longer duration of anesthesia $(67.14 \pm 11.58)$ than the distal group $(53.52 \pm 8.06)$ $(p=0.001)$. No differences were observed in terms of patient satisfaction and complications $(p>0.050)$.

Conclusion: Using the proximal ulnar nerve anesthesia approach was associated with a delayed onset and longer anesthesia than the distal method.

Keywords: anesthesia, ulnar nerve, angiography

\section{INTRODUCTION}

Coronary artery diseases (CADs) and acute coronary syndrome are still the leading cause of mortality among developing and developed countries (1-3). Coronary angiography (CAG) was described by Mason Sones in the early 1960s for the first time and is the most important diagnostic method for patients with CADs and acute coronary accidents (4). Currently, most of the CAGs and percutaneous coronary interventions $(\mathrm{PCI})$ are performed using the trans-femoral approach. This method is effective and beneficial in patients requiring left and right heart catheterization but could also be associated with different complications $(4,5)$. During the past decades, there has been an increasing trend for performing coronary catheterization through the upper limbs (6). Recent studies have indicated that this method is associated with fewer chances of hemorrhage, faster hemostasis, higher patient satisfaction, and also shorter hospital stays than with the femoral approach (7).

Upper limb CAG and $\mathrm{PCl}$ are routinely performed using the trans-radial method, which was introduced by Campeau in 1989 (8). This method has its share of failures depending on the size of the artery, anatomical variation, arterial loops, hypoplasia, and previous arterial harvesting (7). A trans-ulnar method has also been

\section{Farshad Roghani-Dehkordi* (iD \\ Fereshteh Farazandeh** (ID)}

Mohammad Kermani-Alghoraishi* (i)

Alireza Khosravi*** (D)

Departments of Cardiology, and *Interventional Cardiology Research Center, ${ }^{* *}$ Cardiac Rehabilitation Research Center,

***Hypertension Research Center, Cardiovascular Research Institute Isfahan University of Medical Sciences; Isfahan-Iran

Corresponding Author: Mohammad Kermani-Alghoraishi 凹sm.kermani@med.mui.ac.ir

Accepted: June 28, 2021

Available Online Date: December 24, 2021

Cite this article as: Roghani-Dehkordi $F$, Farazandeh F, Kermani-Alghoraishi $M$, Khosravi A. Comparison of pain severity, satisfaction, and complications of proximal and distal forearm anesthesia in patients undergoing trans-palmar coronary angiography. Anatol J Cardiol 2022; 26: 37-42.

DOI: 10.5152/AnatolJCardiol.2021.282 
an effective technique because of the higher diameter of this artery than the radial artery. Studies have also investigated complications of trans-ulnar angiography and have shown that these complications are somehow similar to the trans-radial technique. The term "more distal access" was introduced by Roghani-Dehkordi et al. $(7,9)$ in 2018 to describe approaches distal to the wrist crease, especially distal branch of the ulnar artery as trans-palmar access. They indicated that trans-palmar CAG is safe, feasible, and reliable with lower rate of complications $(7,9)$.

Local anesthesia is an important principle in angiography, which could affect later consequences such as the sense of pain and patient satisfaction (10). Therefore, investigating the best anesthetic method in upper limb angiography has pivotal importance. To the best of our knowledge, no previous studies have evaluated the best anesthetic methods in patients undergoing trans-palmar coronary angiography. Local anesthesia in the distal forearm has a higher chance of complications owing to the close proximity of the ulnar nerve to the vascular and muscular structures. However, local anesthesia in proximal parts of the forearm is associated with greater motor response (11). Therefore, in this study, we aimed to evaluate patient satisfaction, pain severity, and possible complications between local anesthesia through distal and proximal forearm in patients undergoing trans-palmar coronary angiography.

\section{METHODS}

Study participants and design

This was a randomized clinical trial performed in 2019-2020 at the Shahid Chamran Heart Center in Isfahan, Iran. The study population consisted of 60 patients who were candidates for trans-palmar CAG. This study was approved by the Research Committee of Isfahan University of Medical Sciences and was confirmed by the Ethics Committee (Ethics Approval ID: IR.MUI.MED.REC.1398.320). This study is registered in the Iranian Registry of Clinical Trials (IRCT) (https:/ www.irct.ir) with IRCT number IRCT20181228042155N3.

The inclusion criteria were patients who were candidates for trans-palmar CAG and signing the written informed consent to participate in this study. The exclusion criteria were requiring coronary angioplasty, unstable hemodynamics and cardiogenic shock, coagulopathy and anticoagulant use with elevated international normalized ratio (INR), and severe renal failure. On the basis of medical history and simple physical examinations, patients suspected to have Raynaud's syndrome, carpal tunnel syndrome, sensory-motor disorders

\section{HIGHLIGHTS}

- Distal ulnar nerve anesthesia has a faster onset than proximal approach in patients undergoing trans-palmar coronary angiography.

- Proximal ulnar nerve anesthesia is associated with a longer duration of anesthesia for trans-palmar access.

- There was no significant difference in patient satisfaction and complications between the proximal and distal methods. that affect the ulnar and median nerves, chronic tenosynovitis, having deformity of the hand or forearm, ulnar tunnel syndrome (Guyon's channel), and arthritis or joint disease involving the elbow and wrist were also excluded. The study population was collected using the census method during 10 months (September 2019 to June 2020). Demographic data of the patients including age, sex, previous medical diseases, and laboratory data were collected. They were randomized into 2 groups using the sealed envelope technique. The first group underwent trans-palmar CAG using local anesthesia through the distal forearm, and the second group received local anesthesia through the proximal forearm.

In the distal group, subcutaneous lidocaine $2 \%$ (5-7 mL) was injected 1 inch proximal to the pisiform bone and lateral to the flexor carpi ulnaris tendon; whereas in the proximal group, the same dose of lidocaine $2 \%$ was injected in the area 1 to $2 \mathrm{~cm}$ proximal to the internal epicondyle (ulnar sulcus) at an angle of $45^{\circ}$ (Fig. 1). Because the palmar artery is a very spastic artery, sedation was needed. Midazolam 1 to $2 \mathrm{mg}$ and sublingual nitroglycerin $0.4 \mathrm{mg}$ were used. Afterward, a spasmodic intra-arterial cocktail was used, which contained $250 \mathrm{mcg}$ of TNG and $2.5 \mathrm{mg}$ of verapamil. Heparin 2,500 to 5,000 units was used as anticoagulant. Puncture of the arteries was initiated almost 10 to 15 minutes after injections in all the patients. For trans-palmar CAG, the forearm was placed at an angle of $45^{\circ}$, and the hand kept in an extended position (about $20^{\circ}-$ $\left.30^{\circ}\right)$, avoiding hyperextension to prevent arterial collapse. The puncture site was in the medial part of the palmar surface and $1 \mathrm{~cm}$ distal and lateral to the pisiform bone and 1 inch distal to the wrist crease. Anesthesia was evaluated with an insulin syringe needle, and supplementary doses were injected in very small amounts in the puncture site as needed. Angiography started with $21 \mathrm{G}$ needles and 0.018 guidewire under fluoroscopy, and a 6F sheath was used (Fig. 2). Once the procedure was performed, and the sheath was removed, the wrist was hyperextended (up to $90^{\circ}$ ) for arterial hemostasis (9).

\section{Variables}

We evaluated the patients' pain severity using a visual analog scale (VAS) scoring the pain from 0 (least pain) to 10 (worst pain) (12). VAS was measured by the puncture time, just after ending the procedure, 4 hours after procedure, at discharge, and after 1 month. Durations of anesthesia were also measured after CAG by asking the patient and physical examination by sticking the insulin syringe needle into the skin every 10 to 15 minutes. Cardiologists were also asked about the presence of ulnar artery spasm during the procedure. We evaluated the occurrence of hematoma during angiography and in recovery as well as the presence of paresthesia or numbness at the time of discharge. Patient satisfaction was measured using the Patient Satisfaction Checklist for Anesthesia. The scoring of expressions is based on the Likert scale as follows: very dissatisfied (0-1), dissatisfied (2-4), have no opinion (5-6), satisfied (7-8), and very satisfied (9-10).

\section{Statistical analysis}

Statistical analysis was performed using the SPSS version 23 software (SPSS Inc., Chicago, IL, USA). Quantitative variables were reported as mean \pm standard deviation, and 

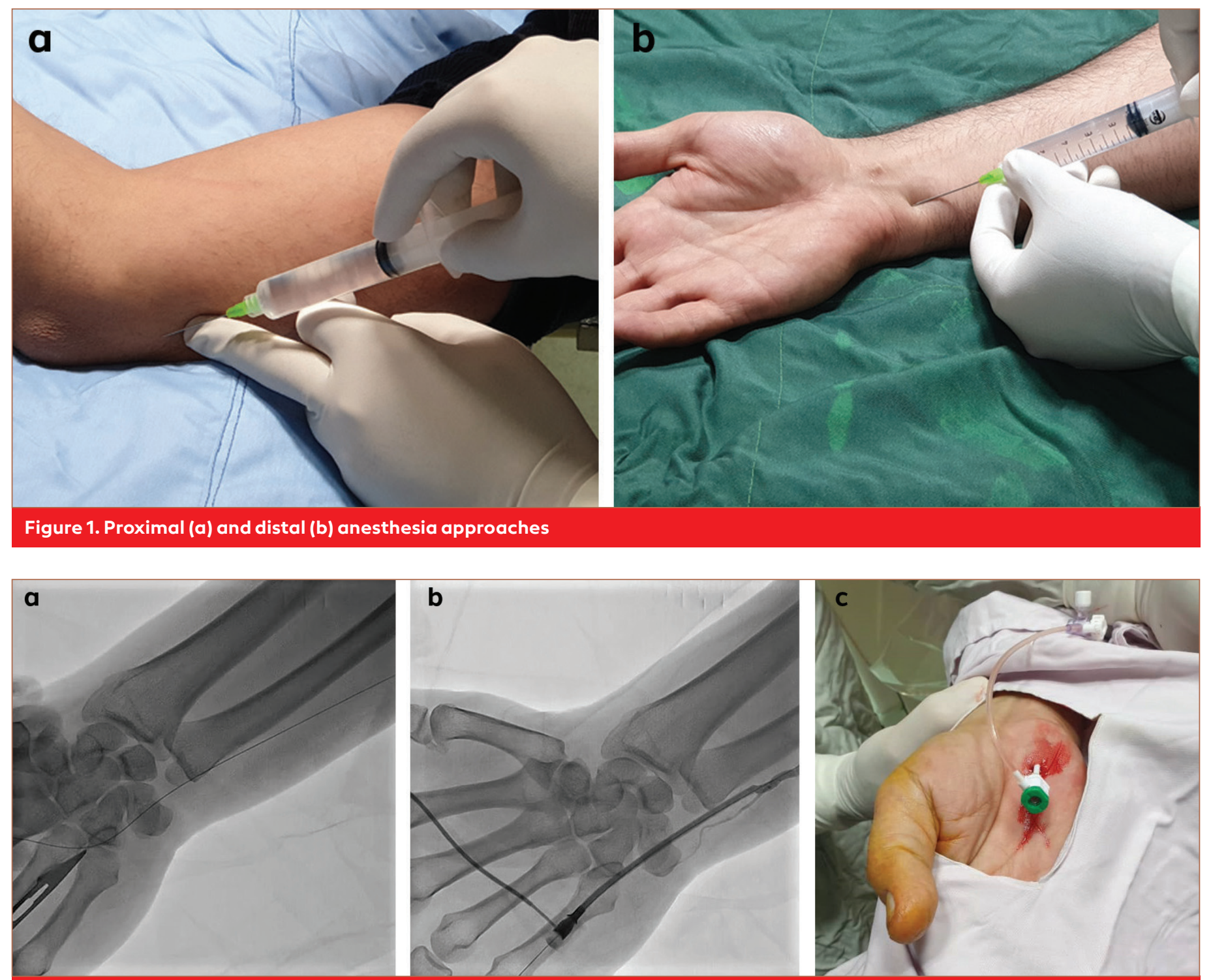

Figure 2. Angiographic depiction of the trans-palmar access (a, b) and cannulation of the distal ulnar artery (palmar artery) (c)

qualitative variables were expressed as number (percentage). The data were in normal distribution; therefore, the variable t test was used to compare the means of continuous variables, and the chi-squared test was used for categorical data. The Friedman test was used to compare the mean VAS measures between the groups. A two-tailed $p$ value $<0.050$ was considered statistically significant.

\section{RESULTS}

A total of 60 patients with successful palmar artery puncture were included in the study. Of note, 2 patients in the proximal approach group and 3 patients in the distal approach group were excluded on the basis of the patient's will and lack of proper cooperation and follow-ups. The Consolidated Standards of Reporting Trial flow diagram is presented in Figure 3.

Data of 55 patients were analyzed. Demographic data of the patients are summarized in Table 1. No significant differences were observed between the two groups in terms of age, sex, and BMI $(p>0.050)$. There were also no significant differ- ences between the 2 groups of patients regarding underlying diseases, such as diabetes, hypertension, and hyperlipidemia; and smoking status, antiplatelet drug consumption, and laboratory data including hemoglobin, creatinine, and platelet count $(p>0.050)$.

We also compared the data regarding the number of puncture attempts, dosage of heparin, time of hospitalization, and need for re-anesthesia. These data showed no significant differences between the 2 groups (Table 2).

According to the results, at the time of puncture, the mean pain severity in the proximal group was significantly higher than the distal group; however, over time and immediately after the procedure and at discharge, the mean pain severity in the proximal group was significantly less than in the distal group ( $p<0.050)$. These data also indicated a significant difference between the 2 groups in the duration of anesthesia. There was also a longer duration of anesthesia in the proximal group (67.14 \pm 11.58 ) than that in the distal group (53.52 \pm 8.06 ) 
Table 1. Basic and laboratory characteristics

\begin{tabular}{|c|c|c|c|}
\hline Variables & Proximal group ( $n=28$ ) & Distal group ( $n=27$ ) & $P$-value \\
\hline Age (mean $\pm S D$ ) & $58.75 \pm 7.49$ & $60.15 \pm 6.70$ & 0.751 \\
\hline Gender (male), n (\%) & $16(57)$ & $18(66)$ & 0.467 \\
\hline $\mathrm{BMI}\left(\mathrm{kg} / \mathrm{m}^{2}\right)($ mean $\pm \mathrm{SD})$ & $29.21 \pm 3.24$ & $29.89 \pm 3.61$ & 0.452 \\
\hline Diabetes mellitus (yes), n (\%) & $8(29)$ & $9(33)$ & 0.702 \\
\hline Hypertension (yes), n (\%) & $15(54)$ & $16(59)$ & 0.670 \\
\hline Hyperlipidemia (yes), n (\%) & $11(39)$ & $11(41)$ & 0.912 \\
\hline Platelets $($ per $/ \mu L)($ mean $\pm S D)$ & $225962.96 \pm 41238.96$ & $247592.59 \pm 50182.94$ & 0.091 \\
\hline Creatinine $(\mathrm{mg} / \mathrm{dL})($ mean $\pm S D)$ & $1.09 \pm 0.21$ & $1.09 \pm 0.22$ & 0.998 \\
\hline Antiplatelet usage, n (\%) & $0.79 \pm 0.41$ & $0.70 \pm 0.46$ & 0.499 \\
\hline
\end{tabular}

BMI - body mass index; SD - standard deviation

\section{Table 2. Procedural data}

\begin{tabular}{|c|c|c|c|}
\hline Variables & $\begin{array}{c}\text { Proximal } \\
\text { group }(n=28)\end{array}$ & $\begin{array}{l}\text { Distal group } \\
\quad(n=27)\end{array}$ & $P$-value \\
\hline $\begin{array}{l}\text { Puncture attempts, } \\
\mathrm{n}(\%)\end{array}$ & $2 \pm 0.72$ & $2.07 \pm 0.65$ & 0.818 \\
\hline $\begin{array}{l}\text { Dosage of heparin } \\
\text { (IU), mean } \pm \text { SD }\end{array}$ & $3482.14 \pm 1417.3$ & $3611.1 \pm 1601.2$ & 0.755 \\
\hline $\begin{array}{l}\text { Hospitalization time } \\
\text { (hour), mean } \pm S D\end{array}$ & $14.79 \pm 5.38$ & $14.69 \pm 4.87$ & 0.901 \\
\hline $\begin{array}{l}\text { Need for } \\
\text { re-anesthesia, n (\%) }\end{array}$ & $12(42.9 \%)$ & $10(\% 37)$ & 0.639 \\
\hline
\end{tabular}

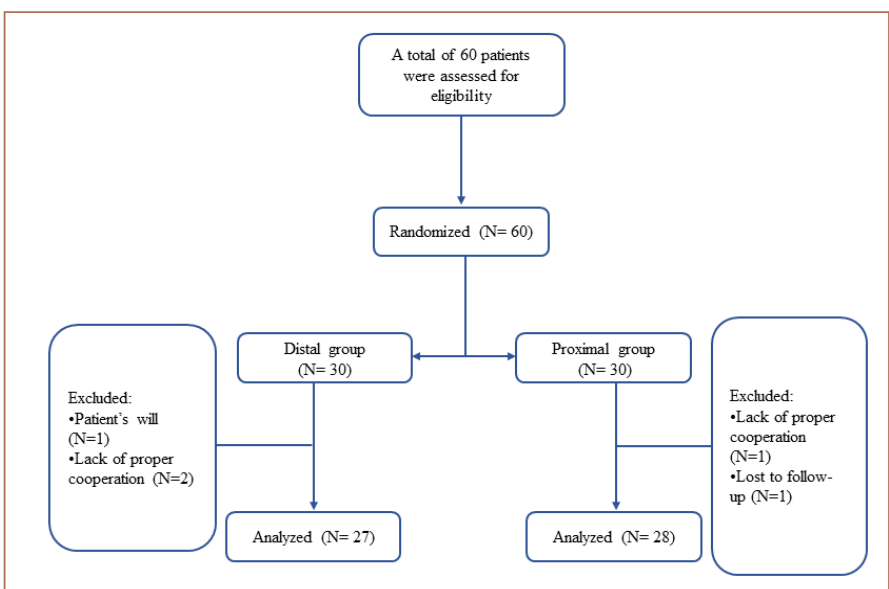

Figure 3. CONSORT diagram

CONSORT - Consolidated Standards of Reporting Trials

$(p=0.001)$. No differences were observed regarding patient satisfaction $(p=0.206)$. These data are summarized in Table 3. Evaluation and comparison of the frequency distribution of complications, including paresthesia and/or numbness of the fingers, hematoma, and ulnar artery spasm in the patients in the distal and proximal groups showed no significant differences among them ( $p>0.050)$ (Table 3).

\section{DISCUSSION}

In this innovative study, we investigated and compared 2 different anesthesia methods for trans-palmar CAG. On the basis of our results, the mean pain severity in the proximal group was significantly higher than in the distal group by the time of puncture; however, over time and immediately after the procedure and at discharge, the mean pain intensity in the proximal group was significantly less than the distal group. There was also a longer duration of anesthesia in the proximal group than in the distal group. These data indicate a longer initiation of anesthesia with the proximal technique in addition to a long-lasting anesthesia. We also observed no significant differences between the groups in terms of complications and procedural data, such as the number of puncture attempts and need for re-anesthesia.

Ulnar nerve block at the level of elbow and wrist are useful methods to supplement a brachial plexus block in hand surgeries and has been previously described $(11,13)$. Per these studies, proximal forearm anesthesia in the ulnar nerve has more motor responses and induce adduction of the hand with or without flexion of the fourth and fifth fingers. Probable complications such as bleeding, hematoma formation, and local anesthetic toxicity were observed more in wrist ulnar nerve block owing to the proximity of the ulnar nerve and ulnar artery. The possibility of tendon trauma by needle tips is also higher in the distal forearm anesthetic injection (13, 14). However, the distal nerve block also has its advantages in that block onset times are faster, and proximal muscle function and limb motor strength are preserved. The prolonged anesthesia in the proximal approach may be because of the later onset of anesthesia and consequently its longer recovery (15-18). However, more comparative studies are needed. We believe that patients who require complex $\mathrm{CAG/PCI}$ or patients undergoing elective angiography could benefit from anesthesia through the proximal forearm owing to the longer duration of anesthesia, in contrast to distal anesthesia which could be useful for faster and shorter intervention cases.

Trans-palmar angiography is a novel technique and is safe, feasible, and associated with a low complication rate and short hospitalization stay (7). Anesthesia is also another important principle in this technique. Roghani-Dehkordi et al. (7) have evaluated 221 patients undergoing angiography and compared snuff box and palmar approaches in these patients. They showed that both the methods were effec- 


\begin{tabular}{|c|c|c|c|c|}
\hline Just after the procedure & $1.29 \pm 0.53$ & $1.78 \pm 0.75$ & 0.008 & \\
\hline 4 hours later & $0.89 \pm 0.56$ & $1.15 \pm 0.86$ & 0.281 & 0.001 \\
\hline Duration of anesthesia & $67.14 \pm 11.58$ & $53.52 \pm 8.06$ & & 0.001 \\
\hline \multicolumn{5}{|l|}{ Satisfaction score } \\
\hline Median ( $25 \%-75 \%$ percentile) & $8(7-9)$ & $8(7-9)$ & & 1.0 \\
\hline Mean $\pm S D$ & $8.11 \pm 0.87$ & $7.74 \pm 1.02$ & & 0.206 \\
\hline Paresthesia/numbness, n (\%) & 1 & 3 & & 0.281 \\
\hline
\end{tabular}

SD - standard deviation; VAS - visual analog scale

tive and associated with appropriate success rates. In this study, patients in the trans-palmar approach received anesthesia through the distal forearm. The main complications of this approach included hand ecchymosis, hematoma of the proximal forearm, and hematoma of the distal arm that were self-limiting in nature with no requirement for further therapy. Another study was performed by Roghani-Dehkordi et al. (9) in 2020 on 106 patients undergoing diagnostic CAG to increase effectiveness and safety and minimize the complications related to trans-palmar angiography. The use of distal ulnar approach in the palmar artery for CAG was also investigated by Valsecchi et al. in 2019 (19). The patients in these studies underwent anesthesia through the distal forearm, which were not associated with any significant complications. Our study results also showed just minor complications, including paresthesia/numbness and hematoma in both the distal and proximal approaches.

\section{Study limitations}

An important point of this study was that the comparison of distal and proximal forearm anesthesia has never been performed before. However, our study had its limitations. First, the sample size was small, and studies with larger sample sizes are recommended. Second, our study was not performed with patients requiring coronary angioplasty. Third, we did not use ultrasound guidance for accurate anesthesia injection puncture. Upper extremity nerve block is made easier and more reliable with the use of ultrasonography guidance (20). Finally, we did not mention the total dose of anesthetics; however, there was very little difference between the injectable doses.

\section{CONCLUSION}

In summary, this study showed that pain severity declined in both the approaches of proximal (elbow site) and distal (wrist site) forearm anesthesia in patients who underwent CAG via trans-palmar access. Pain severity with the proximal anesthesia approach was significantly higher than that in the distal group by the time of puncture; however, over time and immediately after the procedure and at discharge, the mean pain in the proximal group was significantly less than in the distal group. The proximal approach also had longer duration of anesthesia compared that of the distal group. Therefore, proximal local anesthesia of the forearm can be introduced as a novel and alternative route of regional anesthesia during upper extremity coronary catheterizations, especially procedures that require a long time.

Acknowledgments: We thank Mr. Mohammad Amini (Interventional Radiology Technologist at Shahid Chamran Heart Center) for his help in this study.

Conflict of interest: None declared.

Peer-review: Externally peer-reviewed.

Author contributions: Concept - F.R.D., F.F., M.K.A.; Design - F.R.D., F.F., M.K.A., A.K.; Supervision - F.R.D., M.K.A.; Fundings - F.R.D., F.F., M.K.A., A.K.; Materials - F.R.D., M.K.A., A.K.; Data collection \&/or processing - F.R.D., F.F., M.K.A.; Analysis \&/or interpretation F.R.D., F.F., M.K.A.; Literature search - F.F., M.K.A.; Writing - F.R.D., F.F., M.K.A., A.K.; Critical review - F.R.D., F.F., M.K.A, A.K.

\section{REFERENCES}

1. Prabhakaran D, Jeemon P, Roy A. Cardiovascular Diseases in India: Current Epidemiology and Future Directions. Circulation 2016; 133: 1605-20. [Crossref]

2. Roth GA, Huffman MD, Moran AE, Feigin V, Mensah GA, Naghavi $M$, et al. Global and regional patterns in cardiovascular mortality from 1990 to 2013. Circulation 2015; 132: 1667-78. [Crossref]

3. Thygesen K, Alpert JS, Jaffe AS, Chaitman BR, Bax JJ, Morrow DA, et al.; Executive Group on behalf of the Joint European Society of Cardiology (ESC)/American College of Cardiology (ACC)/ American Heart Association (AHA)/World Heart Federation (WHF) Task Force for the Universal Definition of Myocardial Infarction. Fourth Universal Definition of Myocardial Infarction (2018). J Am Coll Cardiol 2018; 72: 2231-64. [Crossref]

4. Bourassa MG. The history of cardiac catheterization. Can J Cardiol 2005; 21: 1011-4.

5. Kolkailah AA, Alreshq RS, Muhammed AM, Zahran ME, El-Wegoud MA, Nabhan AF. Transradial versus transfemoral approach for diagnostic coronary angiography and percutaneous coronary intervention in people with coronary artery disease. Cochrane Database Syst Rev 2018; 2018: CD012318. [Crossref] 
6. Zwaan EM, IJsselmuiden AJ, van Rosmalen J, van Geuns RM, Amoroso G, Moerman E, et al. Rationale and design of the ARCUS: Effects of trAnsRadial perCUtaneouS coronary intervention on upper extremity function. Catheter Cardiovasc Interv 2016; 88: 1036-43. [Crossref]

7. Roghani-Dehkordi F, Hashemifard O, Sadeghi M, Mansouri R, Akbarzadeh $M$, Dehghani $A$, et al. Distal accesses in the hand (two novel techniques) for percutaneous coronary angiography and intervention. ARYA Atheroscler 2018; 14: 95-100.

8. Campeau L. Percutaneous radial artery approach for coronary angiography. Cathet Cardiovasc Diagn 1989; 16: 3-7. [Crossref]

9. Roghani-Dehkordi F, Kasiri R, Kermani-Alghoraishi M, Akbari M. What is the preferred hemostasis method for coronary angiography through the palmar artery access? Cardiovasc Interv Ther 2021; 36: 490-7. [Crossref]

10. McCabe J, Guevara C, Renfroe J, Fattahi T, Salman S, Steinberg B. Quantitative analysis of facial soft tissue perfusion during hypotensive anesthesia using laser-assisted indocyanine green fluorescence angiography. Int J Oral Maxillofac Surg 2018; 47: 465-9. [Crossref]

11. McCahon RA, Bedforth NM. Peripheral nerve block at the elbow and wrist. Contin Educ Anaesth Crit Care Pain 2007; 7: 42-4. [Crossref]

12. Wewers ME, Lowe NK. A critical review of visual analogue scales in the measurement of clinical phenomena. Res Nurs Health 1990; 13: 227-36. [Crossref]

13. Thompson WL, Malchow RJ. Peripheral nerve blocks and anesthesia of the hand. Mil Med 2002; 167: 478-82. [Crossref]
14. Pester JM, Varacallo M. Ulnar Nerve Block Techniques. [Updated 2021 Jul 25]. In: StatPearls [Internet]. Treasure Island (FL): StatPearls Publishing; 2021 Jan-. Available from: URL: https:/ www.ncbi.nlm.nih.gov/books/NBK459208/.

15. Tran De QH, Dugani S, Asenjo JF. Upper Extremity Nerve Blocks, Essentials of Regional Anesthesia. In: Edited by Kaye AD, Urman RD, Vadivelu N. Springer, New York; 2012. p.362-8. [Crossref]

16. Andersen JH, Jaeger P, Grevstad U, Estrup S, Geisler A, Vilhelmsen $F$, et al. Systemic dexmedetomidine is not as efficient as perineural dexmedetomidine in prolonging an ulnar nerve block. Reg Anesth Pain Med 2019; 44: 333-40. [Crossref]

17. Brown DL, Sites BD, Spence BC. Section II: upper extremity blocks. In: Brown DL, Boezaart AP, Galway UA, et al., eds. Atlas of Regional Anesthesia. Philadelphia, PA: Saunders Elsevier; 2010. p.31-88.

18. Botte MJ. Nerve anatomy. In: Doyle, Botte, eds. Surgical Anatomy of the Hand and Upper Extremity. Philadelphia, PA: Lippincott Williams and Wilkins; 2003.p.185-236.

19. Valsecchi O, Vassileva A, Cereda AF. Distal Ulnar Approach in the Palmar Artery for Coronary Angiography and Intervention: Safety, Feasibility, and Reliability in 15 Patients. Journal of the Association for Vascular Access 2019; 24: 51-6. [Crossref]

20. Sehmbi H, Madjdpour C, Shah UJ, Chin KJ. Ultrasound guided distal peripheral nerve block of the upper limb: A technical review. J Anaesthesiol Clin Pharmacol 2015; 31: 296-307. [Crossref] 